\title{
What is the incidence of myocardial necrosis in elective patients discharged on the same day following percutaneous coronary intervention?
}

\author{
I Porto, D J Blackman, D Nicolson, G Niccoli, F Z Kahn, O Ormerod, C Forfar, K Channon, \\ A P Banning
}

Heart 2004;90:1489-1490. doi: 10.1136/hrt.2003.027672

7 i he safety and predictability of percutaneous coronary intervention (PCI) has improved dramatically in the last decade. The increased numbers of patients suitable for the procedures have placed pressure on existing health care systems. Treating patients with chronic stable angina on a day case basis without an overnight stay has several attractions, ${ }^{1}$ but there are some concerns over the safety of this approach.

Troponin I is released rapidly following myocardial necrosis, it is highly sensitive, and may be more specific than other enzymatic markers of cardiac damage. Elevation of cardiac markers, mainly creatine kinase-MB, after both elective percutaneous and surgical revascularisation reflects myocardial necrosis and is associated with increased risk of in-hospital and long term adverse events. ${ }^{2}$

Glycoprotein (Gp) IIb/IIIa inhibitors appear to be particularly beneficial in reducing this complication, ${ }^{3}$ but day case intervention precludes overnight administration of Gp IIb/ IIIa inhibitors.

This audit determined the incidence of serum troponin I elevation after 6-8 hours in patients discharged the same day according to pre-specified clinical, angiographic, and procedural criteria.

\section{METHODS}

Two hundred and twenty nine consecutive patients were admitted for elective day case PCI from January to July 2002. This represents $30 \%$ of the total PCIs in our institution during this period $(\mathrm{n}=762)$.

Predetermined clinical and angiographic inclusion and exclusion criteria were used. Patients had stable angina caused by focal disease and were undergoing elective PCI. Patients aged $>80$ years, with unstable symptoms, peripheral vascular disease, insulin requiring diabetes, heart failure, creatinine $>200 \mathrm{mmol} / \mathrm{l}$, or poor social circumstances were excluded. Angiographic exclusions included left main stem, bifurcation, long diffuse lesions, small vessels $(<3 \mathrm{~mm})$, and vein graft disease. Patients were admitted overnight if a significant procedural complication occurred. Admission was also advised for arterial access site complications, persistent chest pain, ECG abnormality, or if the procedure was prolonged. A 6 French femoral approach was used with $300 \mathrm{mg}$ clopidogrel pre-loading given up to three days before the procedure, prescribed at a pre-clerking visit. Heparin was used at either $5000 \mathrm{U}$ or $70 \mathrm{IU} / \mathrm{kg}$. Sheath removal was accomplished with manual compression once activated clotting time was $<200$ seconds. A minority of obese and hypertensive patients had femoral artery closure devices with Angioseal (St Jude). Mobilisation was allowed after two hours. Patients were discharged after a minimum of six hours observation (range 6-10 hours). Blood for troponin I measurement was drawn just before discharge. Troponin concentrations were not available to the physician making the decision to discharge the patient from hospital.

Troponin I was measured using the Immulite troponin I solid phase chemiluminescent enzyme immunometric assay (Diagnostic Product Corporation, Los Angeles, California, USA). The upper cut off concentrations for normality suggested by the manufacturer were considered positive for the analysis (troponin I concentration $\geqslant 1 \mu \mathrm{g} / \mathrm{l}$ ).

\section{RESULTS}

The procedure was successful in 223 patients (97\%). Six failures occurred: one broken catheter at aortic bifurcation, referred for surgery; and five failed attempts to reopen chronically occluded vessels, discharged same day. Stents were deployed in $91 \%$ of cases. Mean age was 64 years, $77 \%$ were male, and $14 \%$ were non-insulin dependent diabetic patients. Treated vessels were: left anterior descending coronary artery (LAD) in 125 patients (55\%), circumflex coronary artery $(\mathrm{Cx})$ in 48 patients $(21 \%)$, and right coronary artery (RCA) in 56 patients (24\%). A further $15 \%$ of patients had PCI on a second vessel. Mean stent length was $19.6 \mathrm{~mm}$.

Abciximab was used in 22 patients (9.6\%), when judged necessary by the operator. Five patients receiving abciximab were still discharged (the protocol used was a abciximab bolus with six hours infusion protocol and patients went home after a minimum of 12 hours observation).

One hundred and ninety six patients (86\%) underwent same day discharge. These patients had a mean of 1.1 vessels treated with a mean stent length of $18 \mathrm{~mm}$. The mean (SD) radiation dose for the procedure was 3487 (2696) $\mathrm{Gy} / \mathrm{cm}^{2}$. Troponin I was measured in 172 of 196 subjects. The incidence of enzyme elevation in same day discharge patients was $3 \%(5 / 172$ patients $\geqslant 1 \mu \mathrm{g} / \mathrm{l}$; range $1.2-3.1 \mu \mathrm{g} / \mathrm{l})$ Table 1 shows a detailed analysis of these five cases.

Thirty three patients (14\%) were admitted to hospital: in 12 patients abciximab was administered; one patient had angiographic no reflow; 10 patients had residual dissection; one patient was aged $>80$ years; three patients had side branch occlusion; one patient had elevated baseline creatinine; two patients had prolonged ECG changes; and three patients were admitted because of the judgement of the operator. Among the admitted patients, a mean of 1.3 vessels were treated with a mean stent length of $28 \mathrm{~mm}$. The mean radiation dose for the procedure was 5308 (3018) $\mathrm{Gy} / \mathrm{cm}^{2}$ $(\mathrm{p}<0.001 v$ non-admitted patients $)$.

Abbreviations: $C x$, circumflex coronary artery; LAD, left anterior descending coronary artery; RCA, right coronary artery; ISAR REACT, intracoronary stenting and antithrombotic regimen-rapid early action for coronary treatment 
Table 1 Details of the five same day discharge patients with elevated troponin I concentrations at $\geqslant 6$ hours

\begin{tabular}{|c|c|c|c|c|}
\hline & $\begin{array}{l}\text { Troponin I } \\
\text { concentration } \\
(\mu \mathrm{g} / \mathrm{l})\end{array}$ & Reason & $\begin{array}{l}\text { Pre-procedural } \\
\text { criteria satisfied }\end{array}$ & $\begin{array}{l}\text { Same day discharge } \\
\text { criteria satisfied }\end{array}$ \\
\hline Patient 1 & 2.0 & $\begin{array}{l}\text { Single vessel PCI to LAD; } \\
\text { total } 16 \mathrm{~mm} \text { stent }\end{array}$ & Yes & Yes \\
\hline Patient 2 & 1.7 & $\begin{array}{l}\text { Planned stent to proximal LAD } \\
\text { Additional long stent to diffusely } \\
\text { diseased distal LAD } \\
\text { Total } 36 \mathrm{~mm} \text { stent }\end{array}$ & Yes & No \\
\hline Patient 3 & 1.2 & $\begin{array}{l}\text { Planned two vessel } \mathrm{PCl} \\
18 \mathrm{~mm} \text { stent to left } \mathrm{Cx} \text {. Cutting } \\
\text { balloon to LAD in-stent restenosis } \\
\text { and balloon to chronic total } \\
\text { occlusion of distal LAD }\end{array}$ & No & No \\
\hline Patient 4 & 3.1 & $\begin{array}{l}\text { Planned PCI to chronic total } \\
\text { occlusion of RCA } \\
\text { Complicated by hypotension } \\
\text { treated with atropine. Two wall } \\
\text { stents, total stent length } 81 \mathrm{~mm}\end{array}$ & No & No \\
\hline Patient 5 & 1.6 & $\begin{array}{l}\text { Planned single vessel } \mathrm{PCl} \text { for } \\
\text { diffuse LAD disease } \\
\text { Total stent length } 45 \mathrm{~mm}\end{array}$ & No & No \\
\hline
\end{tabular}

\section{DISCUSSION}

These data provide more support for use of a day case PCI strategy in selected low risk patients with stable, noncomplex coronary artery disease. We have previously described the outcome of a larger day case patient population and documented good outcomes up to six months following the procedure. ${ }^{1}$

Previous work has demonstrated that the prognostic value of troponin I elevation is not different between six hour and 24 hour post-PCI samples. ${ }^{4}$ Therefore patients with a normal six hour troponin I concentration are at low risk and can be safely discharged on the same day. There has been considerable debate about the role of Gp IIb/IIIa inhibition in low risk elective patients undergoing PCI. In this study, we have shown that evidence of procedural myocardial necrosis is rare in patients undergoing elective PCI when these clinical, angiographic, and procedural criteria are applied. Our data concurs with recent randomised data from the ISAR REACT study, suggesting no benefit when Gp IIb/IIIa inhibitors were given to low risk patients pre-loaded with clopidogrel.

In this series only 5/196 patients were discharged with evidence of troponin I elevation and a review of these cases demonstrated that four out of five should not have been admitted for the procedure on a day case basis according to our pre-specified criteria. We feel that using these criteria, day case PCI can be used to treat the lowest risk patients; this minimises cost without compromising outcomes. It is essential however to recognise that PCI remains an unpredictable specialty and that admission for overnight care must be available for occasions when the procedural result is not optimal.

\section{Authors' affiliations}

D J Blackman, D Nicolson, F Z Kahn, O Ormerod, C Forfar, K Channon, A P Banning, Department of Cardiology, John Radcliffe Hospital, Oxford, UK

I Porto, G Niccoli, Universita' Cattolica del Sacro Cuore, Roma, Italy

Correspondence to: Dr Adrian P Banning, Department of Cardiology, John Radcliffe Hospital, Headley Way, Headington, Oxford, OX3 9DU, UK; adrian.banning@orh.nhs.uk

Accepted 16 February 2004

\section{REFERENCES}

1 Banning AP, Ormerod OJ, Channon K, et al. Same day discharge following elective percutaneous coronary intervention in patients with stable angina. Heart 2003;89:665.

2 loannidis JP, Karvouni E, Katritsis DG. Mortality risk conferred by small elevations of creatine kinase-MB isoenzyme after percutaneous coronary intervention. J Am Coll Cardiol 2003;42:1406-11.

3 Kini AS, Richard M, Suleman J, et al. Effectiveness of tirofiban, eptifibatide, and abciximab in minimizing myocardial necrosis during percutaneous coronary intervention (TEAM pilot study). Am J Cardiol 2002;90:526-9.

4 Nageh T, Sherwood RA, Harris BM, et al. Cardiac troponin T and I and creatine kinase- $M B$ as markers of myocardial injury and predictors of outcome following percutaneous coronary intervention. Int J Cardiol 2003;92:285-93.

5 Kastrati A, Mehilli J, Schuhlen $\mathrm{H}$, et al. A clinical trial of abciximab in elective percutaneous coronary intervention after pretreatment with clopidogrel. N Engl J Med 2004;350:232-8. 